\title{
Development status of the UV-VIS detector system of SOXS for the ESO-NTT telescope
}

\author{
Rosario Cosentino ${ }^{\mathrm{a}, \mathrm{b}}$, Marcos Hernandez ${ }^{\mathrm{a}}$, Hector Ventura ${ }^{\mathrm{a}}$, Sergio Campana ${ }^{\mathrm{c}}$, Riccardo \\ Claudi $^{\mathrm{d}}$, Pietro Schipani ${ }^{\mathrm{e}}$, Matteo Alivertic ${ }^{\mathrm{c}}$, Andrea Baruffolo ${ }^{\mathrm{d}}$, Sagi Ben-Amif ${ }^{\mathrm{f}, \mathrm{g}}$, Federico \\ Biondi $^{\text {h, Giulio Capasso }}{ }^{\text {e, Francesco D'Alessio }}$, Paolo D’Avanzo ${ }^{\mathrm{c}}$, Ofir Hershkof ${ }^{\mathrm{f}}$, Hanindyo \\ Kuncarayakti $^{\mathrm{j}, \mathrm{k}}$, Marco Landoni ${ }^{\mathrm{c}}$, Matteo Munari ${ }^{\mathrm{b}}$, Giuliano Pignata ${ }^{\mathrm{l}, \mathrm{m}}$, Adam Rubin ${ }^{\mathrm{n}}$, \\ Salvatore Scuderi ${ }^{\mathrm{b}, \mathrm{o}}$, Fabrizio Vitali ${ }^{\mathrm{i}}$, David Young ${ }^{\mathrm{p}}$, Jani Achrén ${ }^{\mathrm{q}}$, José Antonio \\ Araiza-Duran $^{\mathrm{l}, \mathrm{r}}$, Iair Arcavi ${ }^{\mathrm{s}}$, Anna Brucalassi ${ }^{\mathrm{m}, \mathrm{t}}$, Rachel Bruch ${ }^{\mathrm{f}}$, Enrico Cappellaro ${ }^{\mathrm{d}}$, Mirko \\ Colapietro $^{\mathrm{e}}$, Massimo Della Valle ${ }^{\mathrm{e}}$, Marco De Pascale ${ }^{\mathrm{d}}$, Rosario Di Benedetto ${ }^{\mathrm{b}}$, Sergio D'Orsi ${ }^{\mathrm{e}}$, \\ Avishay Gal-Yam , Matteo Genonic ${ }^{\mathrm{c}}$, Jari Kotilainen ${ }^{\mathrm{j}, \mathrm{k}}$, Gianluca Li Causi ${ }^{\mathrm{u}}$, Seppo Mattila ${ }^{\mathrm{j}}$, \\ Michael Rappaport ${ }^{\mathrm{g}}$, Kalyan Radhakrishnan ${ }^{\mathrm{d}}$, Davide Ricci ${ }^{\mathrm{d}}$, Marco Riva ${ }^{\mathrm{c}}$, Bernardo \\ Salasnich $^{\mathrm{d}}$, Stephen Smartt ${ }^{\mathrm{p}}$, Ricardo Zanmar Sanchez ${ }^{\mathrm{b}}$, Maximilian Stritzinger ${ }^{\mathrm{v}}$, Matteo \\ Accardo $^{\mathrm{n}}$, Leander H. Mehrgan ${ }^{\mathrm{n}}$, Derek Ives $^{\mathrm{n}}$, and Josh Hopgood ${ }^{\mathrm{n}}$ \\ ${ }^{a}$ INAF - Fundación Galileo Galilei, Breña Baja, Spain \\ ${ }^{b}$ INAF - Osservatorio Astrofisico di Catania, Catania, Italy \\ ${ }^{\mathrm{c}} \mathrm{INAF}$ - Osservatorio Astronomico di Brera, Merate, Italy \\ ${ }^{\mathrm{d}} \mathrm{INAF}$ - Osservatorio Astronomico di Padova, Padua, Italy \\ eINAF - Osservatorio Astronomico di Capodimonte, Naples, Italy \\ ${ }^{\mathrm{f}}$ Weizmann Institute of Science, Rehovot, Israel \\ 'Harvard-Smithsonian Center for Astrophysics, Cambridge, USA \\ hMax-Planck-Institut für Extraterrestrische Physik, Garching, Germany \\ ${ }^{i}$ INAF - Osservatorio Astronomico di Roma, Rome, Italy \\ jTuorla Observatory, Department of Physics and Astronomy, University of Turku, Turku, \\ Finland \\ ${ }^{\mathrm{k}}$ FINCA - Finnish Centre for Astronomy with ESO, Turku, Finland \\ ${ }^{\text {l} M i l l e n n i u m ~ I n s t i t u t e ~ o f ~ A s t r o p h y s i c s ~(M A S), ~ S a n t i a g o, ~ C h i l e ~}$ \\ ${ }^{m}$ Universidad Andres Bello, Santiago, Chile \\ ${ }^{\mathrm{n}}$ European Southern Observatory, Garching, Germany \\ ${ }^{\circ}$ INAF - Istituto di Astrofisica Spaziale e Fisica Cosmica, Milano, Italy \\ PQueen's University Belfast, Belfast, UK \\ qIncident Angle Oy, Turku, Finland \\ ${ }^{\mathrm{r}}$ Centro de Investigaciones en Optica A. C., León, Mexico \\ sTel Aviv University, Tel Aviv, Israel \\ ${ }^{\mathrm{t}}$ INAF-Osservatorio Astrofisico Arcetri, Firenze, Italy \\ uNAF - Istituto di Astrofisica e Planetologia Spaziali, Rome, Italy \\ vAarhus University, Aarhus, Denmark
}

Further author information: (E-mail: cosentino@tng.iac.es, Telephone: +34 922433642 


\begin{abstract}
SOXS will be the new spectroscopic facility for the ESO NTT telescope able to cover the optical and NIR bands by using two different arms: the UV-VIS $(350-850 \mathrm{~nm})$, and the NIR $(800-2000 \mathrm{~nm})$. In this article, we describe the development status of the visible camera cryostat, the architecture of the acquisition system and the progress in the electronic design. The UV-VIS detector system is based on a CCD detector 44-82 from e2v, a custom detector head, coupled with the ESO continuous flow cryostats (CFC), a custom cooling system, based on a Programmable Logic Controller (PLC), and the New General Controller (NGC) developed by ESO. This paper outlines the development status of the system, describes the design of the different parts that make up the UVVIS arm and is accompanied by a series of information describing the SOXS design solutions in the mechanics and in the electronics parts. The first tests of the detector system with the UV-VIS camera will be shown.
\end{abstract}

(Ref. 1-28).

Keywords: spectrograph, UV-VIS, detector,Control System, CCD

\title{
1. THE UV-VIS DETECTOR SYSTEM
}

The UV-VIS CCD Detector System for SOXS is based on the e2v CCD44-82, the ESO NGC CCD controller unit and an ad hoc designed camera.

\subsection{The Detector}

The $\mathrm{e} 2 \mathrm{v}$ detector is a back illuminated CCD with a $15 \mu$ square pixel and an image area of $30.7 \times 61.4 \mathrm{~mm}$. The high quantum efficiency $(\mathrm{QE})$ in the spectral response of the spectrograph $(350-850 \mathrm{~nm})$ and their geometric characteristics (pixel size and dimensions) make this CCD the most suitable for our instrument.

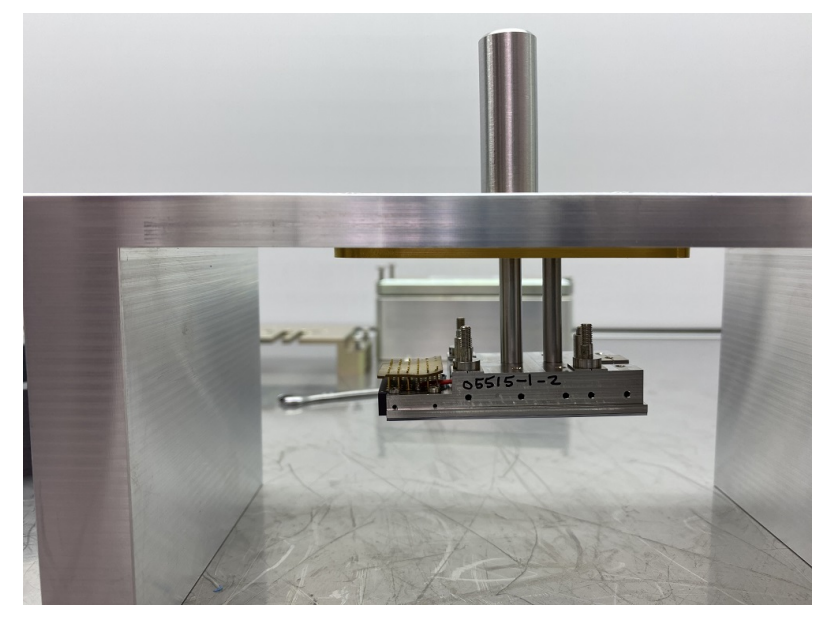

Figure 1. The engineering CCD on the mounting facilities hardware.

\subsection{The NGC controller}

The ESO CCD controller (NGC) is a programmable electronic system for the UV-VIS CCD Detector polarization and readout. The flexibility in the programming of the operative parameters (bias and clock voltages, clock sequences, electronic gain) of the CCD and the features provided for the testing of the signals (programmable test points on the NGC front panel) helps the realization of the test and the optimization of the CCD operative conditions. In addition, the NGC is fully compatible with ESO software and it makes it easier the integration of the SOXS spectrograph in the software NTT environment. 


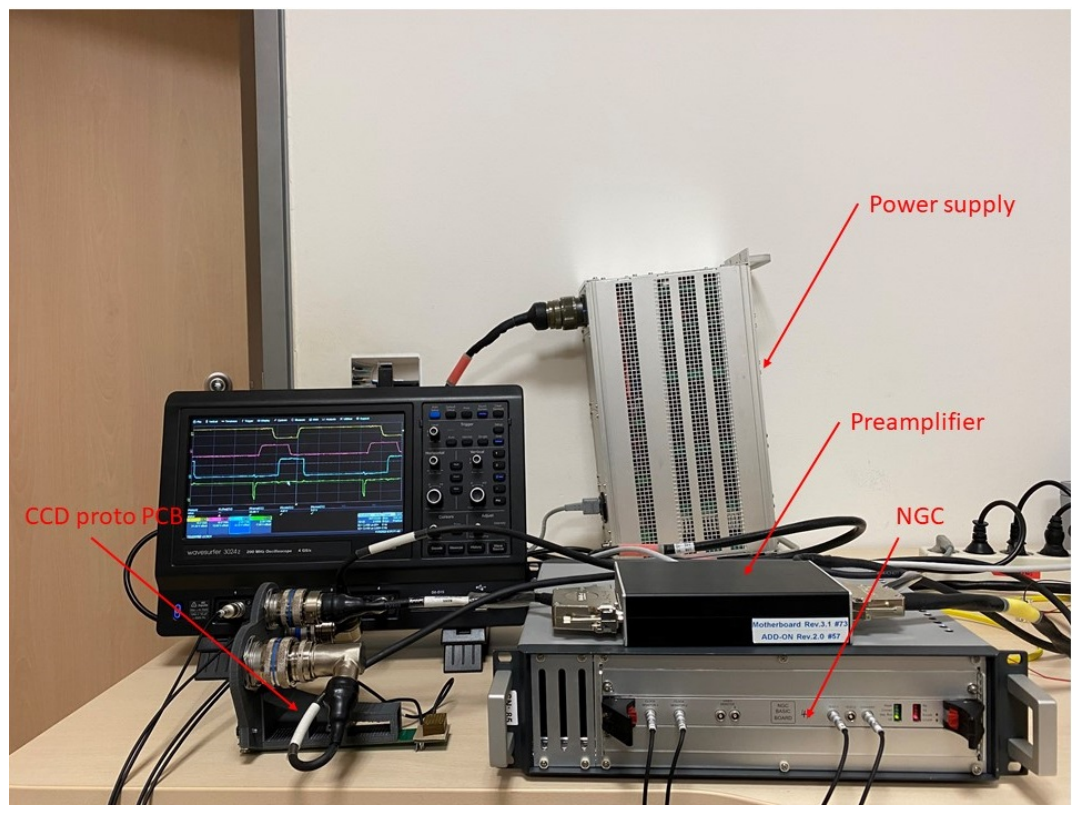

Figure 2. The CCD test bench with the NGC and test facilities.

\subsection{The camera housing}

The camera is made up by the cryogenic part, based on the Continuous Flow Cryostat (CFC) coupled with the UV-VIS camera and the wiring for the detector and for the temperature sensors and control. The mechanic design of the camera is optimized to be mounted inside the reduced dimension of the UV-VIS spectrograph with the consequence of a reduced space inside the camera for the electronic connections. The reduced space inside the camera led to the development of an optimized Printed Citcuit Board (PCB), based on flex technology, for the connection of the CCD with the front-end electronics (Figure 4).

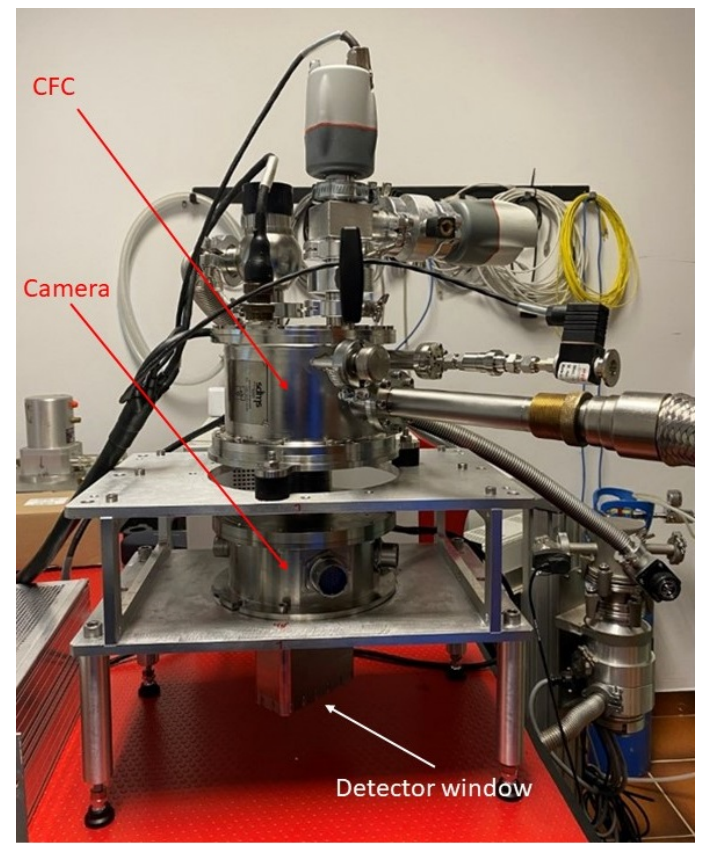

Figure 3. UV-VIS camera coupled with CFC. 


\subsection{The internal wiring}

For the internal wiring we adopted a solution based on a flex cable for the CCD connection, a coaxial cable for the video signals and a twisted vacuum wires for the temperature reading and control.

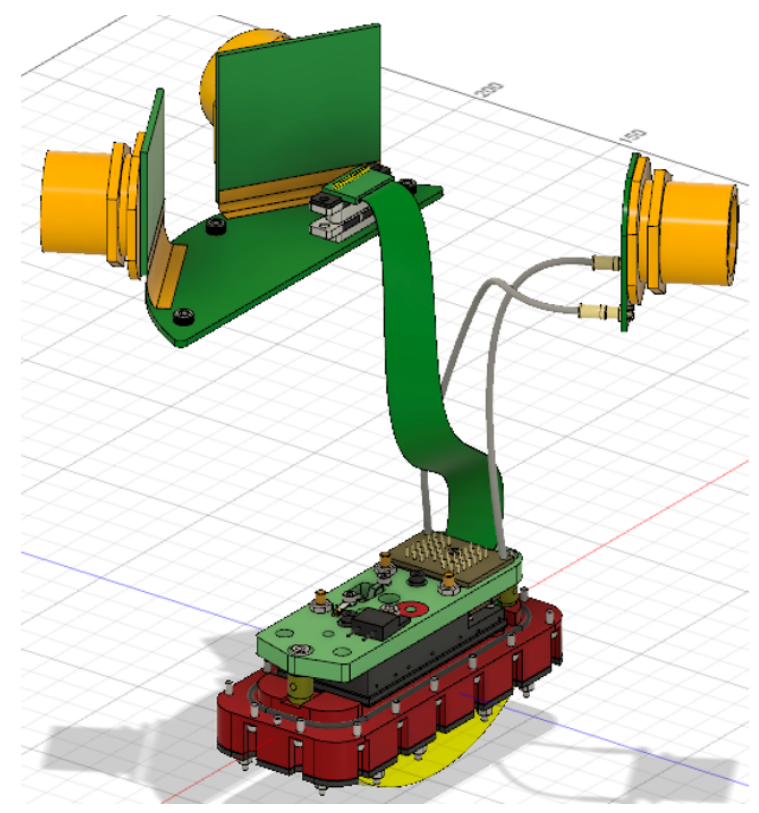

Figure 4. design of the CCD flex boards.

\section{UV-VIS LABORATORY TESTS}

The first test, to optimize the connections and minimize the noise, was done with a prototype PCB (Figure 9) and with a laboratory CCD housing (Figure 10). In these test we measured the CCD clocks, the bias and the video signals.

\subsection{Electronic tests}

The measures of the clocks signals shown a correct temporization of the phases and the good quality of the signals on the Zero Insertion Force (ZIF) socket of the CCD. Figure 5 and Figure 6 shown the CCD phases measured in the CCD board (on the ZIF socket).

The preamplifier was tested with a signal generator at different frequency to test the programmable bandwidth settings and the corresponding electronic gains (Figure 7). 


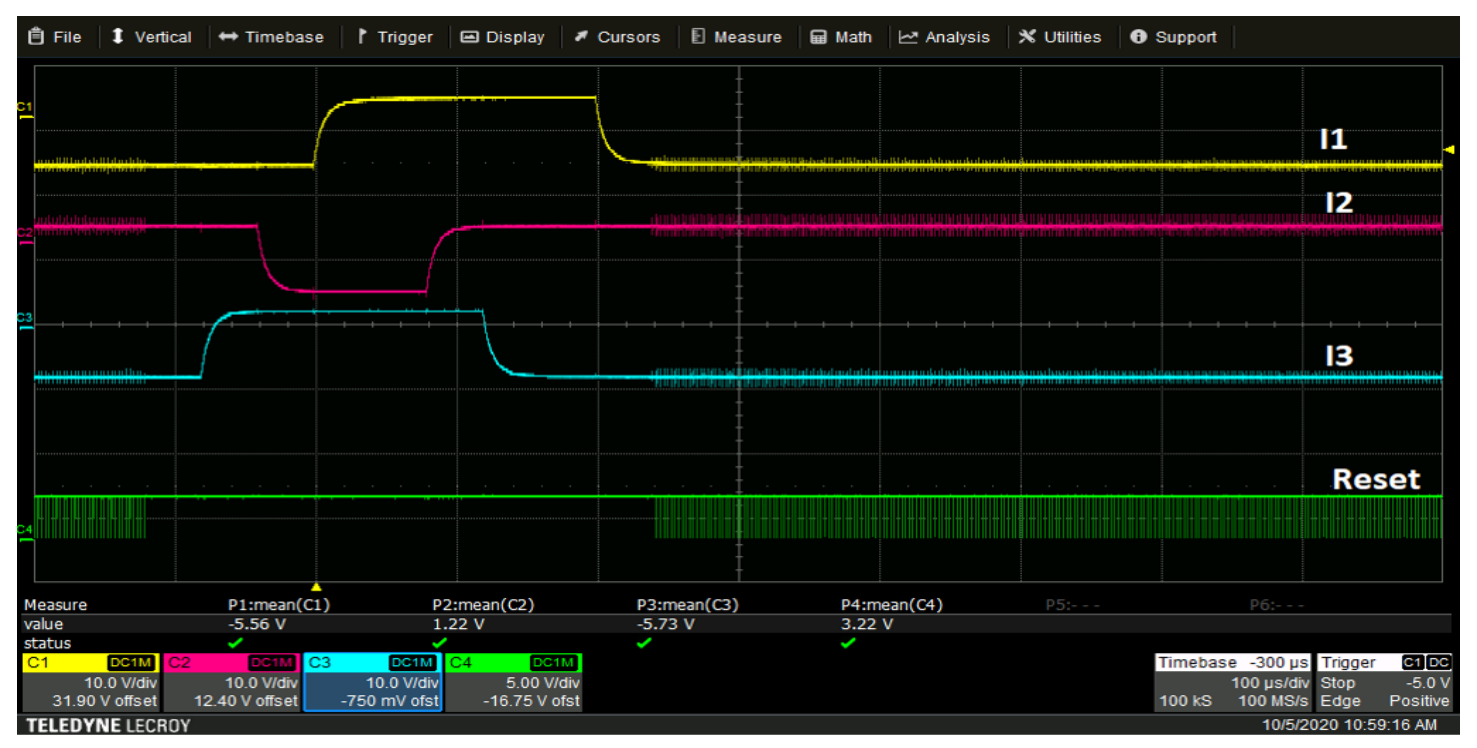

Figure 5. CCD vertical phases

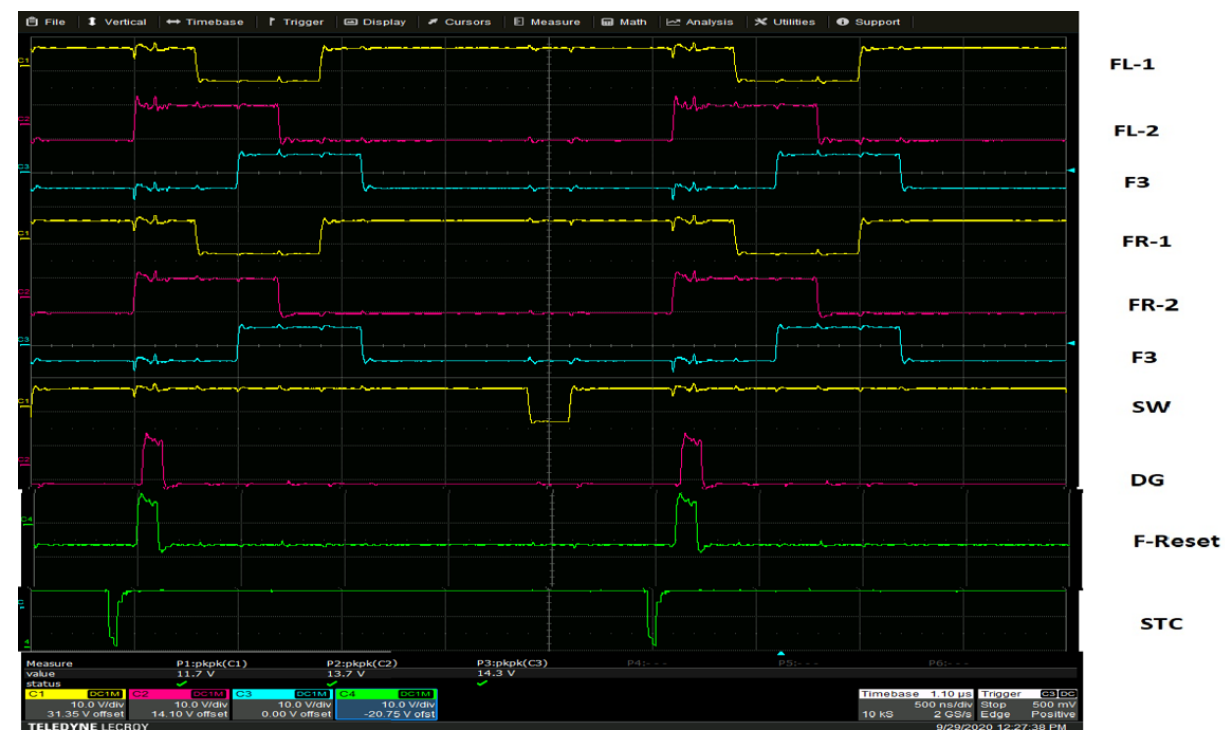

Figure 6. CCD horizontal phases and control timing. 


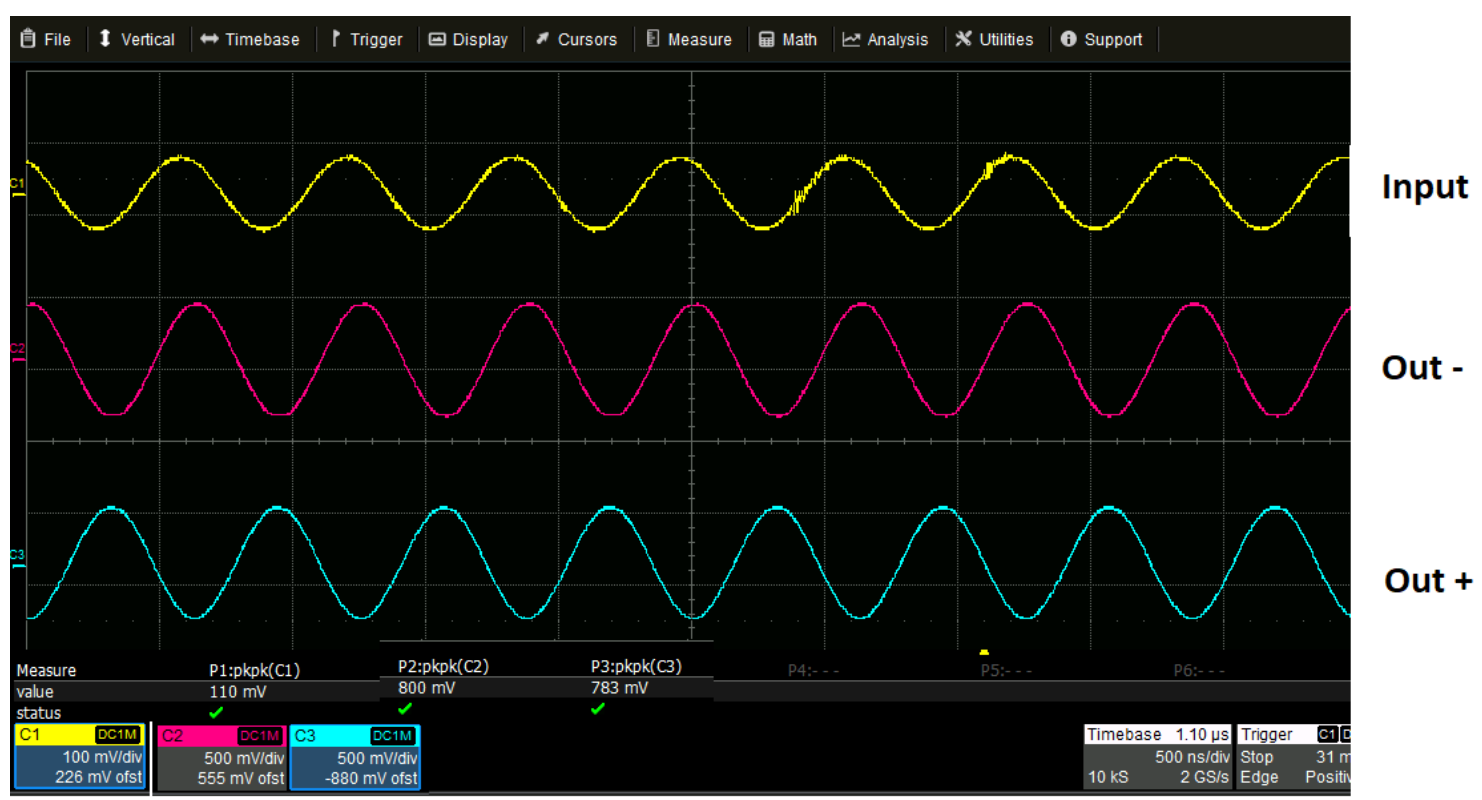

Figure 7. Preamplifier test with an external signal.

\subsection{Detector tests}

The engineering CCD was tested with the test bench shown in Figure 2. We chose the buffered CCD output $\mathrm{OP}$ and the preamplifier input as shown in Figure 8.

\section{Detail of FET Buffer}

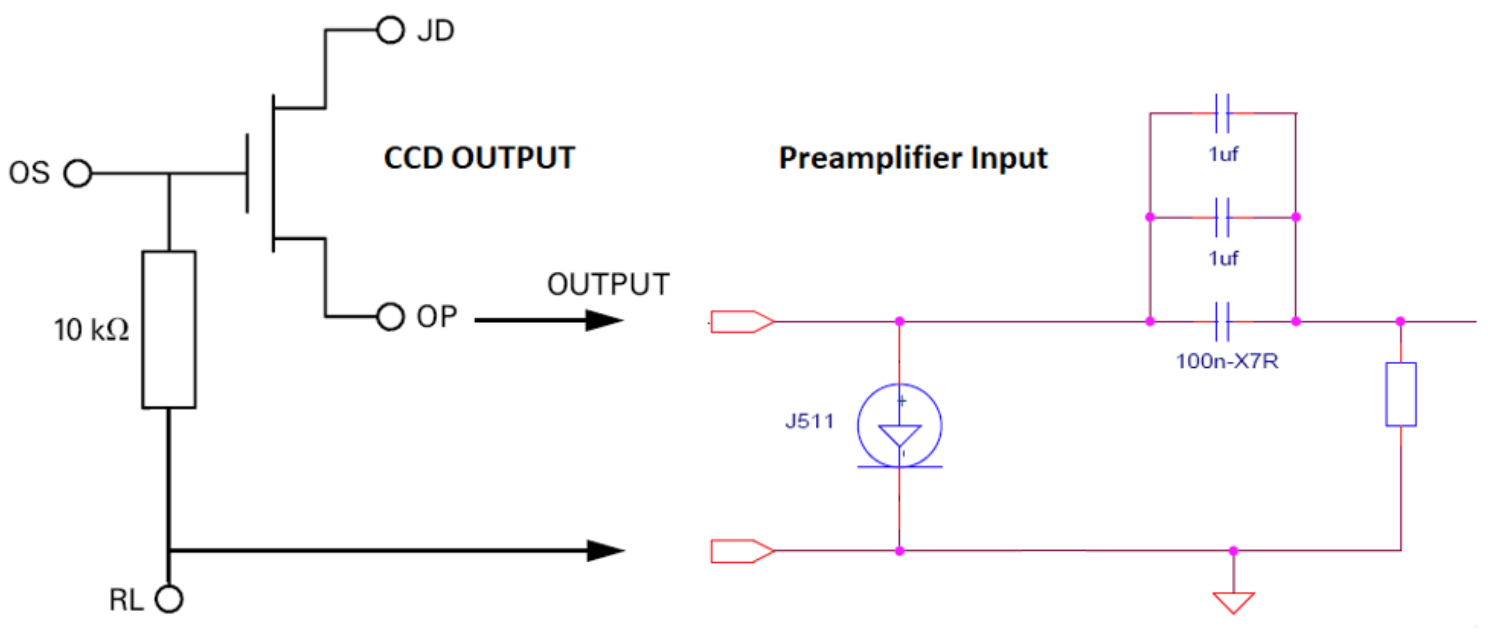

Figure 8. CCD output and preamplifier input. 


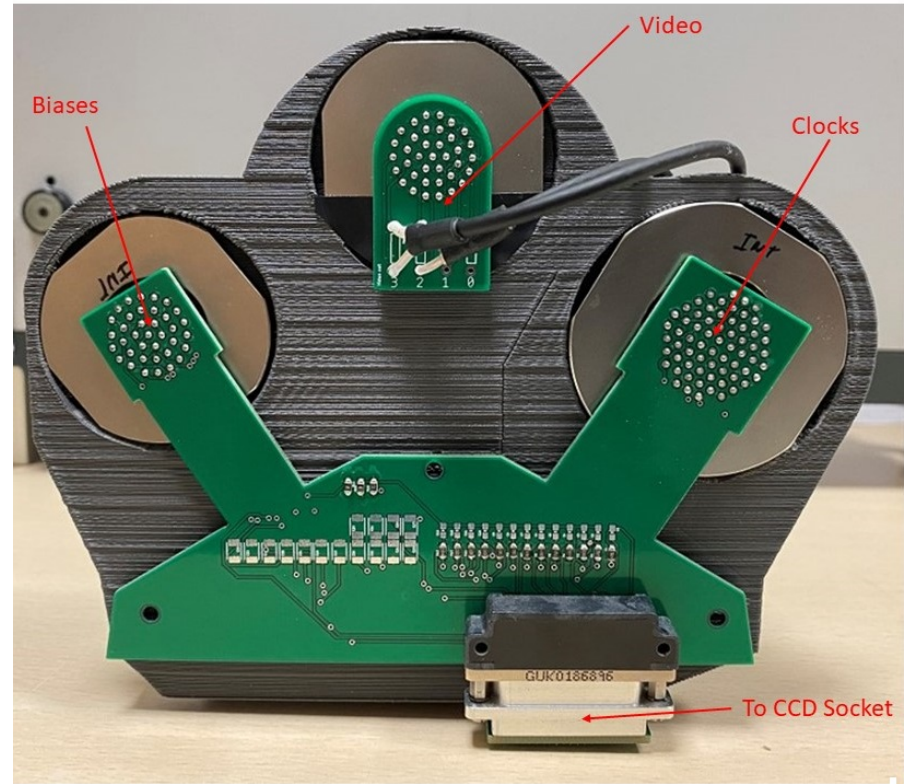

Figure 9. CCD board prototype.
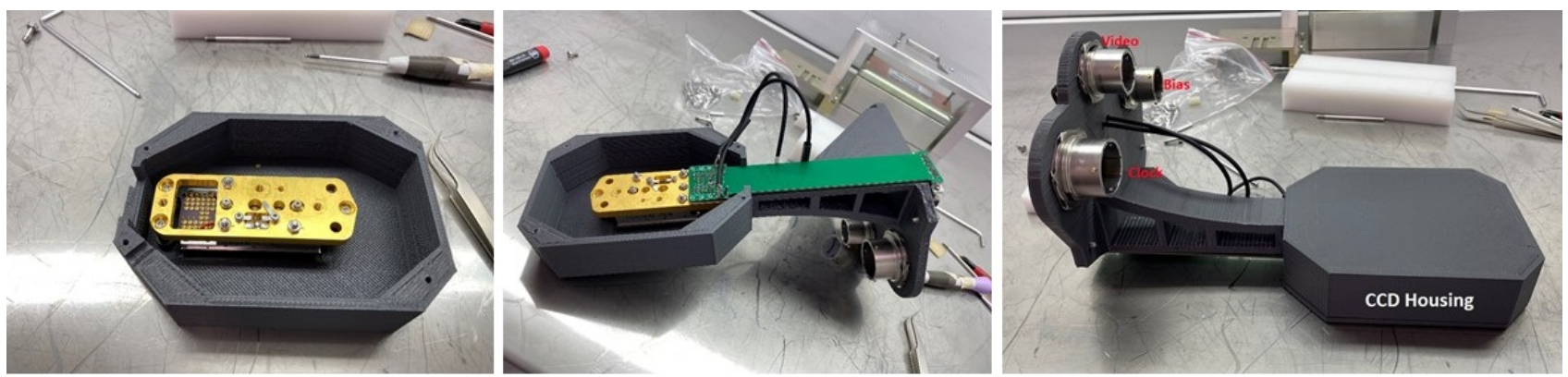

Figure 10. CCD test bench (for CCD warm tests

The tests have been done with an engineering CCD and at room temperature. In this condition is possible to verify if the acquisition system works as expected, because the CCD image has to show the overscan and the expected thermal signal. We have adjusted the video offsets and the gain and we acquired three images, one for each readout mode. In Figure 11 are shown the CCD images, acquired in the three readouts mode available (Left-Right, Left and Right). In these images the overscan is clearly visible and the images shows the thermic electrons. In this engineering CCD seems that the serial register is broken, because in the images and with more evidence in the horizontal cuts ( Figure 12) part of the CCD did not show the thermal signal. 


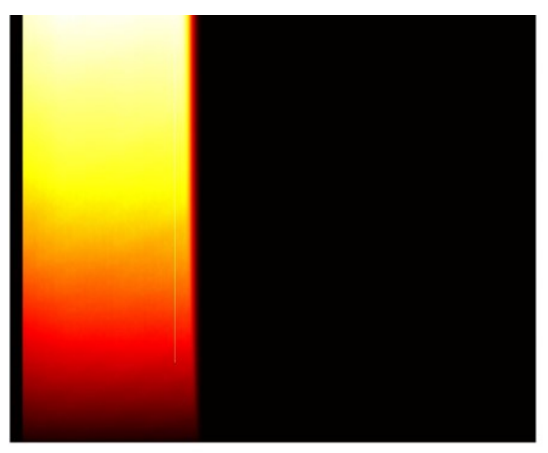

Left

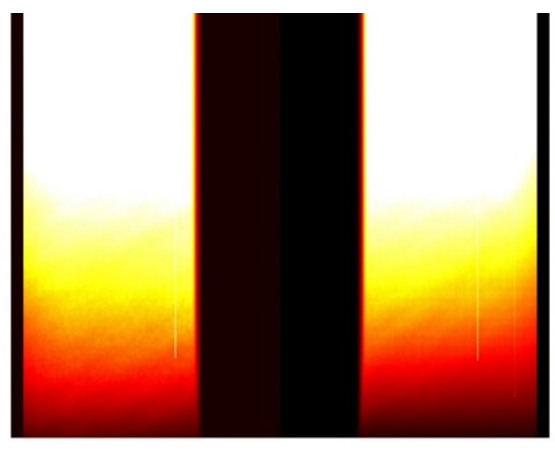

Left-Right

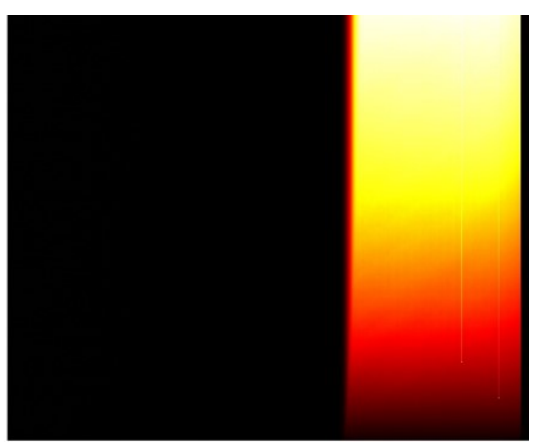

Right

Figure 11. CCD images (room temperature).

The acquired images shown that the thermal signal increase with a gradient trend as expected (Figure

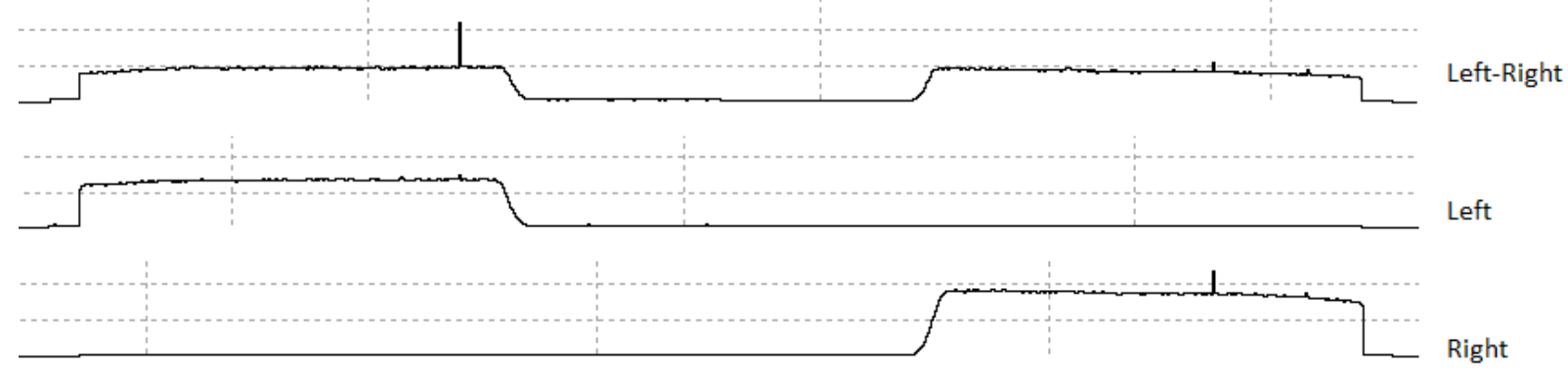

Figure 12. CCD vertical cuts.

More optimizations, at cryogenic temperature, will be done with the SOXS UV-VIS camera, the final flex $\mathrm{PCB}$ and the scientific CCD.

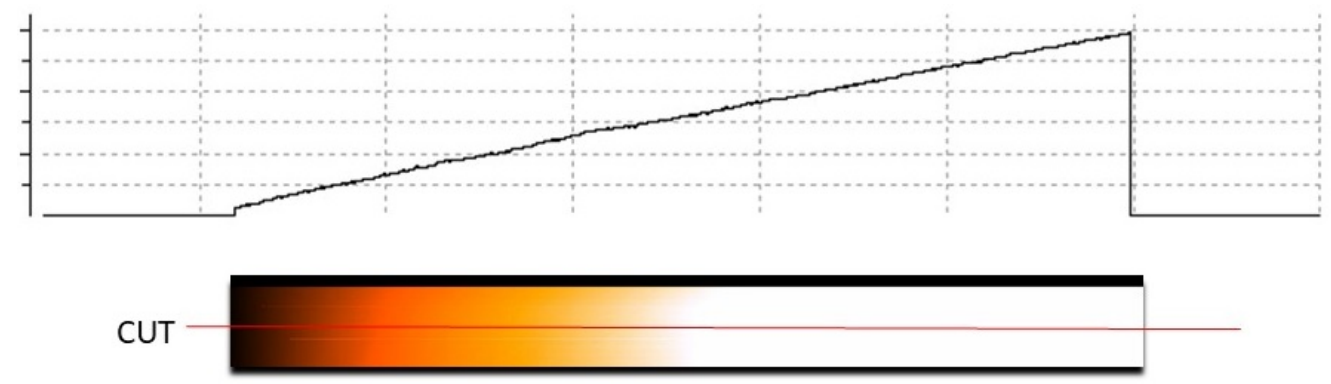

Figure 13. CCD horizontal cuts.

\subsection{Vacuum tests}

The preliminary vacuum test was done with the dummy CCD and without the CCD wiring. Leak test are foreseen for mid December 2020.

\subsection{Cryogenic tests}

The cryogenic test was done with satisfactory results. The CFC shown a low LN2 consumption in a 10 days continuous cooling (less than $0.5 \mathrm{l} / \mathrm{hr}$ ) and the equilibrium CCD temperature reach the expected values. 


\subsection{Conclusions}

The tests on the prototype PCBs with the engineering CCD shown that the acquisition system works as expected. The next step will be the final test at cryogenic temperature with the scientific CCD in order to optimize the CCD settings and reach the optimum performance in term of thermic stability of the detector, minimum readout noise and data throughput.

\section{ACKNOWLEDGMENTS}

A special acknowledgement to the European Southern Observatory for the support provided and for the availability to share its knowledge and to allow the use of the ESO laboratories in Garching.

\section{REFERENCES}

[1] Schipani, P. et al., "The new SOXS instrument for the ESO NTT," in [Proc. SPIE 9908, 990841], (2016).

[2] Cosentino, R. et al., "The vis detector system of SOXS," in [Proc. SPIE 107022J], (2018).

[3] Aliverti, M. et al., "The mechanical design of SOXS for the NTT," in [Proc. SPIE 10702], (2018).

[4] Biondi, F. et al., "The assembly integration and test activities for the new SOXS instrument at NTT," in [Proc. SPIE 10702], (2018).

[5] Brucalassi, A. et al., "The acquisition camera system for SOXS at NTT," in [Proc. SPIE 10702], (2018).

[6] Capasso, G. et al., "SOXS control electronics design," in [Proc. SPIE 10702], (2018).

[7] Claudi, R. et al., "The common path of SOXS (Son Of X-Shooter)," in [Proc. SPIE 10702], (2018).

[8] Zanmar Sánchez, R. et al., "Optical design of the SOXS spectrograph for ESO NTT," in [Proc. SPIE 10702], (2018).

[9] Schipani, P. et al., "SOXS: a wide band spectrograph to follow up the transients," in [Proc. SPIE 10702], (2018).

[10] Ricci, D. et al., "Architecture of the SOXS instrument control software," in [Proc. SPIE 10702], (2018).

[11] Rubin, A. et al., "MITS: the multi-imaging the transient spectrograph for SOXS," in [Proc. SPIE 10702], (2018).

[12] Vitali, F. et al., "The NIR spectrograph for the new soxs instrument at the ESO-NTT," in [Proc. SPIE 10702], (2018).

[13] Leander, M. et al., "State-of-the-art detector controller for eso instruments," in [SDW 2013 Florence], (2013).

[14] Aliverti, M. et al., "Manufacturing, integration, and mechanical verification of SOXS," in [Proc. SPIE 11447-137], (2020).

[15] Biondi, F. et al., "The aiv strategy of the common path of SOXS," in [Proc. SPIE 11447-60], (2020).

[16] Brucalassi, A. et al., "Final design and development status of the acquisition and guiding system for SOXS," in [Proc. SPIE 11447-80], (2020).

[17] Claudi, R. et al., "Operational modes and efficiency of SOXS," in [Proc. SPIE 11447-99], (2020).

[18] Colapietro, M. et al., "Progress and tests on the instrument control electronics for SOXS," in [Proc. SPIE 11452-89], (2020).

[19] Genoni, M. et al., "SOXS end-to-end simulator: Development and applications for pipeline design," in [Proc. SPIE 11450-47], (2020).

[20] Kuncarayakti, H. et al., "Design and development of the SOXS calibration unit," in [Proc. SPIE 11447-45], (2020).

[21] Landoni, M. et al., "The SOXS scheduler for remote operation at lasilla: Concept and design," in [Proc. SPIE 11452-36], (2020).

[22] Ricci, D. et al., "Development status of the SOXS instrument control software," in [Proc. SPIE 11452-112], (2020).

[23] Rubin, A. et al., "Progress on the uv-vis arm of SOXS," in [Proc. SPIE 11447-36], (2020).

[24] Zanmar Sánchez, R. et al., "SOXS: Effects on optical performances due to gravity flexures, temperature variations, and subsystems alignment," in [Proc. SPIE 11447-70], (2020). 
[25] Scuderi, S. et al., "The vacuum and cryogenic system of the SOXS spectrograph," in [Proc. SPIE 11451-209], (2020).

[26] Schipani, P. et al., "Development status of the SOXS spectrograph for the ESO-NTT telescope," in [Proc. SPIE 11447-8], (2020).

[27] Vitali, F. et al., "The development status of the NIR spectrograph for the new soxs instrument at the NTT," in [Proc. SPIE 11447-90], (2020).

[28] Young, D. et al., "The SOXS data reduction pipeline," in [Proc. SPIE 11452-98], (2020). 